\title{
Chapter 118 \\ Report on Global Environment \\ Competitiveness of Saudi Arabia
}

Saudi Arabia is the largest state in Western Asia by land area, Its total area is 2,149.7 thousand square kilometers, it is bordered by Jordan and Iraq to the north and northeast, Kuwait, Qatar, Bahrain and the United Arab Emirates to the east, Oman to the southeast, and Yemen to the south. The Red Sea lies to its west, and the Persian Gulf lies to

\begin{tabular}{|l|}
\hline Score: \\
\hline 51.33 \\
\hline Rank: \\
\hline 54 \\
\hline
\end{tabular}
the east. Saudi Arabia has a desert climate with extremely high daytime temperatures and a sharp temperature drop at night. It covers $2,149.7$ thousand of square kilometers and has a population of 28.08 million. Its GDP reaches $\$ 576.82$ billion in 2011 . Through the index system and evaluation model, the environment competitiveness index of Saudi Arabia ranks at 54 in 133 countries.

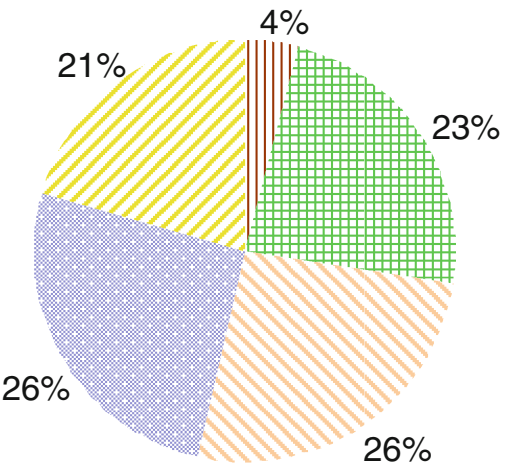

III Resource Environment Competitiveness

\# Ecological Environment Competitiveness

Environment Carrying Competitiveness

- Environment Management Competitiveness

Fig. 118.1 Contribution Environment Harmony of sub-index to GEC

Competitiveness 


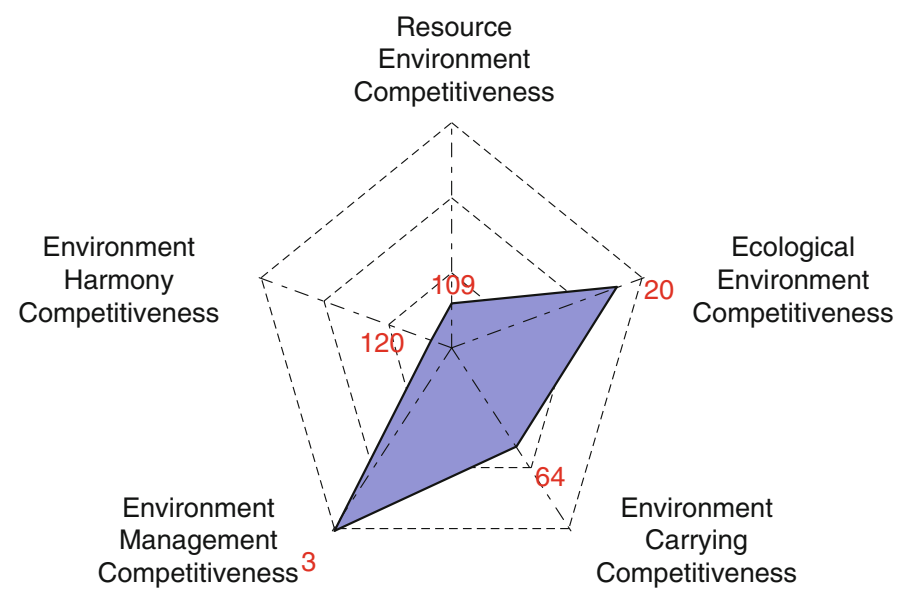

Fig. 118.2 Rank of sub-index of GEC

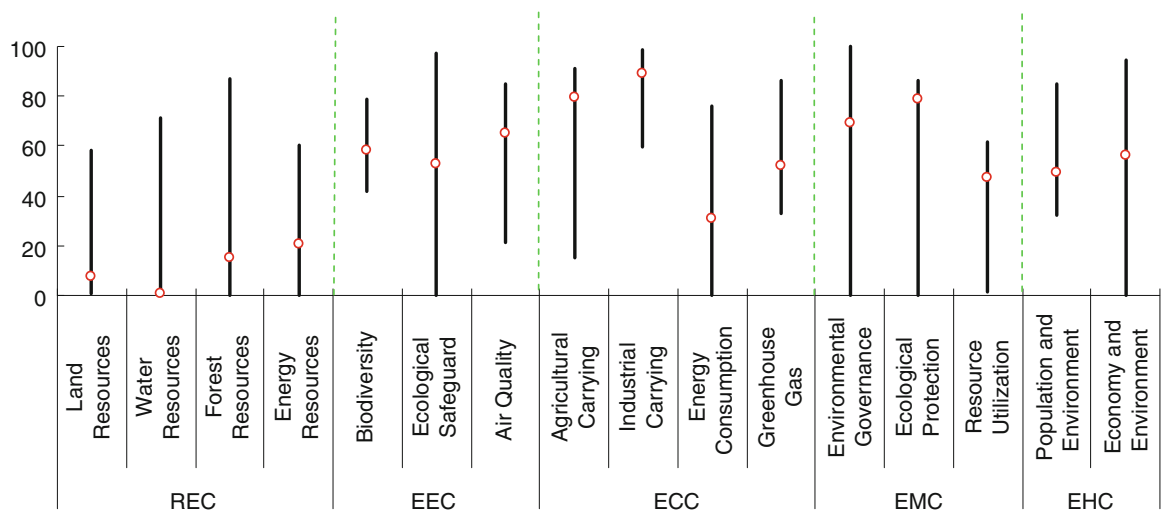

Fig. 118.3 Score and rank of the pillars of GEC

Table 118.1 Score and rank of all indicators of GEC

\begin{tabular}{|c|c|c|c|c|c|}
\hline Indicators & Score & Rank & Indicators & Score & Rank \\
\hline $\begin{array}{l}1 \text { Resource Environment } \\
\text { Competitiveness }\end{array}$ & 11.02 & 109 & $\begin{array}{l}\text { Total internal renewable } \\
\text { water resources }\end{array}$ & 0.06 & 130 \\
\hline 1.1 Land Resources & 7.75 & 102 & 1.3 Forest Resources & 15.29 & 121 \\
\hline Land area per capita & 13.78 & 17 & Growing stock in forest & 50.02 & 109 \\
\hline $\begin{array}{l}\text { Percentage of arable land } \\
\text { to total land area }\end{array}$ & 2.28 & 122 & $\begin{array}{l}\text { and other wooded land } \\
\text { Proportion of land area }\end{array}$ & 0.53 & 125 \\
\hline Arable land per capita & 5.18 & 93 & covered by forest & & \\
\hline 1.2 Water Resources & 0.54 & 131 & Forest area per capita & 0.24 & 114 \\
\hline Surface water & 0.01 & 125 & 1.4 Energy Resources & 20.83 & 12 \\
\hline Annual precipitation & 2.00 & 128 & Fossil energy & 25.99 & 7 \\
\hline Groundwater & 0.11 & 124 & Energy production & 19.80 & 7 \\
\hline
\end{tabular}


Table 118.1 (continued)

\begin{tabular}{|c|c|c|c|c|c|}
\hline Indicators & Score & Rank & Indicators & Score & Rank \\
\hline \multirow{3}{*}{$\begin{array}{l}\text { Proportion of combustible } \\
\text { renewable and waste } \\
\text { to total energy } \\
\text { consumption }\end{array}$} & 0.00 & 118 & 3.3 Energy Consumption & 30.95 & 108 \\
\hline & & & $\begin{array}{l}\text { Energy consumption per } \\
\text { unit of land area }\end{array}$ & 99.84 & 54 \\
\hline & & & Ratio of clean energy & 0.00 & 116 \\
\hline \multirow{2}{*}{$\begin{array}{l}\text { Net energy imports of the } \\
\text { energy consumption }\end{array}$} & 40.65 & 12 & consumption & & \\
\hline & & & Elasticity of energy & 13.71 & 81 \\
\hline \multirow{2}{*}{$\begin{array}{l}2 \text { Ecological Environment } \\
\text { Competitiveness }\end{array}$} & 59.37 & 20 & consumption & & \\
\hline & & & Elasticity of electric power & 10.27 & 83 \\
\hline 2.1 Biodiversity & 58.10 & 65 & consumption & & \\
\hline Threatened fish species & 89.15 & 62 & 3.4 Greenhouse Gas & 51.72 & 125 \\
\hline $\begin{array}{l}\text { Threatened mammal } \\
\text { species }\end{array}$ & 95.11 & 49 & $\begin{array}{l}\text { Growth rate of } \mathrm{CO}_{2} \\
\text { emissions }\end{array}$ & 41.23 & 103 \\
\hline \multirow{3}{*}{$\begin{array}{l}\text { Threatened plant species } \\
\text { GEF benefits index for } \\
\text { biodiversity }\end{array}$} & 99.82 & 40 & Growth rate of Methane & 47.79 & 119 \\
\hline & 3.20 & 61 & emissions & & \\
\hline & & & $\mathrm{CO}_{2}$ emissions per unit of & 99.77 & 69 \\
\hline 2.2 Ecological Safeguard & 52.62 & 12 & land area & & \\
\hline \multirow{2}{*}{$\begin{array}{l}\text { Terrestrial protected areas } \\
\text { Marine protected areas }\end{array}$} & 84.78 & 5 & $\mathrm{CO}_{2}$ emissions per unit of & 28.59 & 105 \\
\hline & 4.38 & 44 & energy consumption & & \\
\hline \multirow{2}{*}{$\begin{array}{l}\text { 2.3 Air Quality } \\
\text { Inhalable particles (PM10) }\end{array}$} & 65.38 & 54 & 4 Environment Management & 66.31 & 3 \\
\hline & 29.93 & 127 & Competitiveness & & \\
\hline \multirow{3}{*}{$\begin{array}{l}\text { Particulate matter (PM2.5) } \\
\text { Index of indoor air } \\
\quad \text { pollution }\end{array}$} & 69.03 & 108 & 4.1 Environmental & 69.10 & 107 \\
\hline & 100.00 & 1 & Governance & & \\
\hline & 100.00 & & Agricultural chemicals & 100.00 & 1 \\
\hline \multirow{2}{*}{$\begin{array}{l}\text { Nitrogen oxides emission } \\
\text { Sulfur dioxide emission }\end{array}$} & 67.62 & 73 & regulation & & \\
\hline & 36.31 & 117 & Percentage of the rural & 0.00 & 122 \\
\hline $\begin{array}{l}3 \text { Environment Carrying } \\
\text { Competitiveness }\end{array}$ & 67.14 & 64 & $\begin{array}{l}\text { population with access } \\
\text { to an improved water }\end{array}$ & & \\
\hline \multirow{2}{*}{$\begin{array}{l}\text { 3.1 Agricultural Carrying } \\
\text { Cereal yield per unit of } \\
\text { arable land }\end{array}$} & 79.57 & 12 & $\begin{array}{l}\text { source } \\
\text { Percentag the urban }\end{array}$ & & \\
\hline & 59.24 & 17 & $\begin{array}{l}\text { Percentage of the urban } \\
\text { population with access }\end{array}$ & 97.00 & 80 \\
\hline \multirow{2}{*}{$\begin{array}{l}\text { Fertilizer consumption per } \\
\text { unit of arable land }\end{array}$} & 96.46 & 53 & $\begin{array}{l}\text { to an improved water } \\
\text { source }\end{array}$ & & \\
\hline & & & 4.2 Ecological Protection & 78.46 & 2 \\
\hline \multirow{2}{*}{$\begin{array}{l}\text { Annual freshwater } \\
\text { withdrawals for } \\
\text { agriculture per unit } \\
\text { of arable land }\end{array}$} & 89.11 & 111 & $\begin{array}{l}\text { Area of plantation and } \\
\text { afforestation }\end{array}$ & N/A & N/A \\
\hline & & & Biome protect & 100.00 & 1 \\
\hline 3.2 Industrial Carrying & 89.11 & 88 & Overfishing of fishing & 56.93 & 43 \\
\hline $\begin{array}{l}\text { Net exports of goods as a } \\
\text { percentage of GDP }\end{array}$ & 61.29 & 114 & $\begin{array}{l}\text { resources } \\
4.3 \text { Resource Utilization }\end{array}$ & 47.32 & 21 \\
\hline \multirow{2}{*}{$\begin{array}{l}\text { Electric power } \\
\text { consumption per unit of } \\
\text { value added of industry }\end{array}$} & 95.46 & 30 & $\begin{array}{l}\text { Utilization rate of water } \\
\text { resources }\end{array}$ & 38.27 & 3 \\
\hline & & & Percentage of total internal & 54.55 & 82 \\
\hline \multirow{2}{*}{$\begin{array}{l}\mathrm{SO}_{2} \text { emissions per unit of } \\
\text { value added of industry } \\
\text { Annual freshwater } \\
\text { withdrawals for } \\
\text { industry per value } \\
\text { added of industry }\end{array}$} & 99.87 & 49 & $\begin{array}{l}\text { renewable water } \\
\text { resources to total water }\end{array}$ & & \\
\hline & 99.83 & 11 & $\begin{array}{l}\text { resources } \\
\text { Percentage of agricultural } \\
\text { land to total land area }\end{array}$ & 95.36 & 4 \\
\hline
\end{tabular}


Table 118.1 (continued)

\begin{tabular}{|c|c|c|c|c|c|}
\hline Indicators & Score & Rank & Indicators & Score & Rank \\
\hline \multirow{3}{*}{$\begin{array}{l}\text { Percentage of fossil fuel } \\
\text { energy consumption } \\
\text { to total energy } \\
\text { consumption }\end{array}$} & \multirow[t]{3}{*}{1.12} & \multirow[t]{3}{*}{121} & $\mathrm{SO}_{2}$ emissions per capita & 75.71 & 117 \\
\hline & & & $\mathrm{CO}_{2}$ emissions per capita & 56.03 & 126 \\
\hline & & & $\begin{array}{l}\text { Energy consumption per } \\
\quad \text { capita }\end{array}$ & 52.39 & 116 \\
\hline $\begin{array}{l}5 \text { Environment Harmony } \\
\text { Competitiveness }\end{array}$ & 52.79 & 120 & $\begin{array}{l}5.2 \text { Economy and } \\
\text { Environment }\end{array}$ & 56.34 & 97 \\
\hline $\begin{array}{l}\text { 5.1 Population and } \\
\text { Environment }\end{array}$ & 49.24 & 127 & $\begin{array}{l}\text { Land resource utilization } \\
\text { efficiency }\end{array}$ & 0.08 & 77 \\
\hline $\begin{array}{l}\text { Percentage of population } \\
\text { with access to }\end{array}$ & N/A & N/A & $\begin{array}{l}\text { Sulfur dioxide emissions } \\
\text { per unit of GDP }\end{array}$ & 88.82 & 92 \\
\hline $\begin{array}{l}\text { Improved sanitation } \\
\text { facilities }\end{array}$ & & & $\begin{array}{l}\text { Carbon dioxide emissions } \\
\text { per unit of GDP }\end{array}$ & 62.82 & 116 \\
\hline $\begin{array}{l}\text { Motor vehicles per } 1,000 \\
\text { people }\end{array}$ & 58.77 & 95 & $\begin{array}{l}\text { Energy consumption per } \\
\text { unit of GDP }\end{array}$ & 73.64 & 95 \\
\hline $\begin{array}{l}\text { Renewable internal } \\
\text { freshwater resources } \\
\text { per capita }\end{array}$ & 0.10 & 128 & & & \\
\hline
\end{tabular}

Table 118.2 Rank distribution of the individual indicators of GEC

\begin{tabular}{lllllll}
\hline & $\begin{array}{l}\text { Number } \\
\text { of the } \\
\text { individual } \\
\text { indicators }\end{array}$ & $\begin{array}{l}\text { Rank } \\
1-10\end{array}$ & $\begin{array}{l}\text { Rank } \\
11-30\end{array}$ & $\begin{array}{l}\text { Rank } \\
31-60\end{array}$ & $\begin{array}{l}\text { Rank } \\
61-100\end{array}$ & $\begin{array}{l}\text { Rank } \\
101-133\end{array}$ \\
\hline Sub-index & 14 & 2 & 2 & 0 & 0 & 10 \\
\hline $\begin{array}{c}\text { Resource Environment } \\
\text { Competitiveness }\end{array}$ & 11 & 2 & 1 & 3 & 3 & 2 \\
$\begin{array}{c}\text { Ecological Environment } \\
\quad \text { Competitiveness }\end{array}$ & 15 & 0 & 3 & 3 & 3 & 6 \\
$\begin{array}{c}\text { Environment Carrying } \\
\quad \text { Competitiveness }\end{array}$ & 10 & 5 & 1 & 0 & 1 & 2 \\
$\begin{array}{c}\text { Environment Management } \\
\quad \text { Competitiveness }\end{array}$ & 10 & 0 & 0 & 0 & 4 & 5 \\
$\begin{array}{c}\text { Environment Harmony } \\
\text { Competitiveness }\end{array}$ & 60 & 9 & 7 & 6 & 11 & 25 \\
\hline \begin{tabular}{c} 
Total \\
\hline
\end{tabular} & & & & & & \\
\hline
\end{tabular}

Open Access This chapter is distributed under the terms of the Creative Commons Attribution Noncommercial License, which permits any noncommercial use, distribution, and reproduction in any medium, provided the original author(s) and source are credited. 